Alter

Revue de phénoménologie

26 | 2018

La nature

\title{
Mythe (s) de l'intériorité et phénoménologie de la nature
}

Élodie Boublil

\section{OpenEdition}

1 Journals

Édition électronique

URL : http://journals.openedition.org/alter/592

DOI : $10.4000 /$ alter.592

ISSN : 2558-7927

Éditeur :

Association ALTER, Archives Husserl (CNRS-UMR 8547)

\section{Édition imprimée}

Date de publication : 31 décembre 2018

Pagination : 85-102

ISBN : 978-2-9550449-4-0

ISSN : $1249-8947$

\section{Référence électronique}

Élodie Boublil, « Mythe (s) de l'intériorité et phénoménologie de la nature », Alter [En ligne], 26 | 2018, mis en ligne le 31 décembre 2019, consulté le 23 mars 2020. URL : http://journals.openedition.org/ alter/592 ; DOI : https://doi.org/10.4000/alter.592 


\title{
MYTHE(S) DE L'INTÉRIORITÉ ET PHÉNOMÉNOLOGIE DE LA NATURE
}

\author{
Élodie Boublil
}

\begin{abstract}
Bien que, cela étant, un abîme incommensurable soit installé entre le domaine du concept de la nature - le sensible - et le domaine du concept de la liberté - le suprasensible - au point que, du premier au second (donc par l'intermédiaire de l'usage théorique de la raison), nul passage n'est possible, tout à fait comme s'il s'agissait de mondes differents, dont le premier ne peut avoir sur le second aucune influence, celui-ci doit pourtant avoir une influence sur celui-là, autrement dit, le concept de liberté doit rendre effectif dans le monde sensible la fin indiquée par ses lois; et il faut nécessairement, par conséquent, que la nature puisse être pensée de façon telle que la légalité de sa forme s'accorde pour le moins avec la possibilité des fins qui doivent être mises en œuvre en elle, selon les lois de la liberté ${ }^{1}$
\end{abstract}

Le rappel de cette perspective kantienne sur la nature, et plus précisément sur l'articulation des concepts de nature et de liberté, a pour vocation de souligner que le passage recherché par Kant entre la " nature » et la "liberté », séparées au premier chef par un "abîme incommensurable ", fut établi grâce au jugement réfléchissant, et grâce à l'idée d'une foi morale dans la nature rationnelle de l'homme, capable de s'arracher aux inclinations sensibles, pathologiques, pour s'élever à la dimension d'être raisonnable, agissant en vue du Souverain Bien. Autrement dit, l'interrogation sur la nature - chez Descartes comme chez Kant - est solidaire d'une interrogation sur la liberté de $l^{\prime}$ homme. La référence au "mythe de l'intériorité $»^{2}$ dans le titre donné à cet article - selon lequel la sphère de la représentation associée à l'agir pratique serait dissociable du monde naturel et humain où il se

\footnotetext{
${ }^{1}$ E. Kant, Critique de la faculté de juger, trad. A. Renaut, Paris, Garnier-Flammarion, p. 154.

2 Titre emprunté à l'ouvrage de J. Bouveresse, Le mythe de l'intériorité. Expérience, signification et langage privé chez Wittgenstein, Paris, Éditions de Minuit, 1976.
} 
déploie - vise à rouvrir cette interrogation concernant les rapports entre la nature et la liberté, dans un contexte phénoménologique. Cet essai met en perspective la conception merleau-pontienne de la nature qui se construit en opposition aux théories kantienne, sartrienne, voire husserlienne de la liberté, avec la conception ricœurienne de la liberté qui s'appuie sur une reprise critique du concept de nature - notamment de nature humaine - dans sa Philosophie de la volontés, et dans la dernière ontologie suggérée par la conclusion de Soi-même comme un $a^{a}$ tre $^{4}$. Loin d'évacuer la question du sujet ou du « métaphysique dans l'homme ", nous chercherons à montrer que la confrontation des interrogations phénoménologiques et herméneutiques du concept de nature proposées à la fois par Merleau-Ponty et par Ricœur aboutissent à une nouvelle topographie de la liberté - entée sur l'être-au-monde de la subjectivité et sur sa relation à une nature qui devient alors l'autre nom de la corporéité et du vivant. Ouvrant la Phénoménologie de la perception par une référence explicite à la Critique de la faculté de juger de Kant, Merleau-Ponty semble faire de l'intentionnalité opérante (fungierende) le point de passage permettant l'ancrage dans le monde naturel et humain d'une liberté conçue comme praxis immanente à l'expression des corps :

Husserl reprend la Critique du Jugement quand il parle d'une téléologie de la conscience. Il ne s'agit pas de doubler la conscience humaine d'une pensée absolue qui, du dehors, lui assignerait des fins. Il s'agit de reconnaître la conscience elle-même comme projet du monde, destinée à un monde qu'elle n'embrasse ni ne possède, mais vers lequel elle ne cesse de se diriger, - et le monde comme cet individu pré-objectif dont l'unité impérieuse prescrit à la conscience son but ${ }^{5}$.

Les développements ultérieurs de Merleau-Ponty, jusqu'aux notes du Visible et l'Invisible, chercheront à penser cette " unité impérieuse " et immanente au monde, sans recourir à une téléologie extrinsèque à son déploiement. Chez Ricœur, il s'agira de penser l'unité immanente à l'agir humain, sans recourir à une téléologie intrinsèque qui ignorerait l'involontaire qui surgit à même la corporéité. Ainsi, la voie phénoménologico-ontologique de Merleau-Ponty et la voie herméneuticomorale de Ricœur répondent toutes deux à un désir de reformulation de la dichotomie posée entre l'intériorité et l'extériorité (l'«abîme incommensurable»), reformulation qui passe alors par une reprise

\footnotetext{
${ }^{3}$ P. Ricœur, Philosophie de la volonté 1, le volontaire et l'involontaire, Paris, Seuil, 1949 ; Philosophie de la volonté 2, finitude et culpabilité, Paris, Seuil, 1960.

${ }^{4}$ P. Ricœur, «Vers quelle ontologie ? », Soi-même comme un autre, Paris, Seuil, 1990, p. 345-411.

${ }^{5}$ M. Merleau-Ponty, Phénoménologie de la perception, avant-propos, Paris, Gallimard, 2002, p. xiii.
} 
phénoménologique du concept de nature, élaborée à partir des descriptions de la chair ou de l'affectivité.

Merleau-Ponty s'achemine vers une ontologie phénoménologique de la nature, devenant endo-ontologie de la chair du monde ${ }^{6}$, en s'appuyant sur la déconstruction d'un mythe de l'intériorité ${ }^{7}$ qui émanciperait la conscience transcendantale de son enracinement charnel et historique. Son approche de la nature comme " être sauvage ${ }^{8}$ reconduit le «sens » à son origine antéprédicative, faisant ainsi du monde l'espace topologique naturel à partir duquel se dessinent et s'instituent les trajectoires historiques de la liberté. La foi perceptive et la plasticité expressive qui se déploient à même le monde naturel épousent les mouvements d'un "être de porosité " ${ }^{9}$ et de latence, et révèlent un logos endiathetos, selon les réflexions du Visible et l'Invisible. Dans la pensée de Merleau-Ponty, la refonte du concept de nature est ainsi fondée sur une approche esthésiologique de l'intériorité - non de l'intériorité d'une conscience séparée, mais de l'intériorité propre à l'être charnel lui-même et qui révèle la portée interrogative et signifiante de la vie. Comme le souligne Françoise Dastur : «la pensée de Merleau-Ponty, surtout dans sa dernière période, est une pensée de la structure vivante pour laquelle l'intériorité ne renvoie plus à un sujet clos sur lui-même, mais devient la dimension d'un être qui en perdant sa positivité en vient à se confondre avec le mouvement même de l'expérience $»^{10}$.

\footnotetext{
${ }^{6} \mathrm{Cf}$ E. de Saint Aubert, Vers une ontologie indirecte, sources et enjeux critiques de l'appel à l'ontologie chez Merleau-Ponty, Paris, Vrin, 2006, p. 138 et sq. Voir aussi : La nature ou le monde du silence, Paris, Hermann, 2008.

7 Dans l'avant-propos de la Phénoménologie de la perception, Merleau-Ponty désigne explicitement l'intériorité augustinienne comme lieu de la méprise concernant l'être au monde de la subjectivité : «L'analyse réflexive croit suivre en sens inverse le chemin d'une constitution préalable et rejoindre dans "l'homme intérieur" comme dit Saint Augustin, un pouvoir constituant qui a toujours été en lui. Ainsi la réflexion s'emporte elle-même et se replace dans une subjectivité invulnérable, en deçà de l'être et du temps », op.cit., p. iv. Mais un tel propos est là aussi à nuancer car comme l'explique Jean-Louis Chrétien : « Le chemin vers l'intérieur n'est donc pas la perte de l'immense, mais la plongée dans une immensité plus déconcertante encore, car sa démesure est en moi, elle est moi. Le privilège augustinien de l'intériorité ne réside pas en ce qu'elle exclut l'extériorité, mais en ce qu'elle l'inclut et l'excède », in La joie spacieuse, Paris, Editions de Minuit, 2007, p. 37.

8 « Ce que je veux faire, c'est restituer le monde comme sens d'Être absolument différent du "représenté", à savoir comme l'Être vertical qu'aucune des "représentations" n'épuise et que toutes "atteignent", l'Être sauvage. », M. Merleau-Ponty, Le visible et l'invisible, note de mai 1960, Paris, Gallimard, 2004, p. 301.

${ }_{9}^{9}$ Comme l'indique Pierre Rodrigo, la voie la plus prometteuse pour la phénoménologie pourrait bien être celle que Maurice Merleau-Ponty envisageait d'explorer dans Le visible et l'invisible : celle d'une ontologie non positive, d'un "être de porosité", ou encore d'un "être brut" ou "sauvage" se donnant sans l'exigence de remplissement ultime des esquisses ", in L'intentionnalité créatrice, Problèmes de phénoménologie et d'esthétique, Paris, Vrin, 2009, p. 19.

${ }^{10}$ F. Dastur, "Merleau-Ponty et la pensée du dedans », in Merleau-Ponty, phénoménologie et expériences, M. Richir et É. Tassin (dir.), Grenoble, Jérôme Millon, 1992, p. 44.
} 
Abolissant les dichotomies de la métaphysique classique, MerleauPonty amorce une philosophie de l'appartenance du sujet au monde fondée sur cette intériorité de l'être à lui-même.

Dans la pensée de Ricœur, le mouvement de l'interrogation est inversé. La refonte du concept de liberté s'appuie sur une approche mythologique ou poétique du concept de nature qui substitue l'idée de polarité à la porosité de l'être merleau-pontien. Le mythe de la nature qui transparaît dans les analyses sur l'involontaire et sur la fragilité fait signe vers une phénoménologie de l'intériorité qui préfigure l'herméneutique de l'altérité et les analyses ultimes de Ricœur sur l'homme capable et sur l'ipséité d'un sujet qui se sait à la fois « agissant et souffrant $»^{11}$. Ricœur déploie alors en filigrane une anthropologie philosophique de la liberté qui met l'accent sur les médiations et met en lumière «l'intermédiaire »12 (metaxu) qui relie l'ancrage naturel et corporel de l'intentionnalité affective au projet libre et créateur de la volonté.

Nous commencerons par analyser les implications de la définition merleau-pontienne de la nature, selon laquelle « est nature ce qui a un sens $»^{13}$. Une telle assertion rature la dialectique du pour-soi et de l'ensoi, et initie une ontologie phénoménologique de la nature qui donne à penser non l'intériorité close d'un sujet qui aurait à rejoindre le monde, mais l'intimité ouverte d'une chair désirante. Puis, en nous appuyant sur un texte de Strasser, intitulé "Réhabilitation de l'intériorité. Réflexions sur la dernière philosophie de Merleau-Ponty », nous analyserons les enjeux de la redéfinition du concept de " nature humaine » à l'œuvre dans les analyses de Ricœur sur l'affectivité. L'intentionnalité que dévoile Ricœur, à travers ce qu'on pourrait nommer une " phénoménologie de l'intériorité » qui évalue tout ce qui résiste en elle, ne met pas au jour les différentes strates du monde naturel. Elle ressaisit plutôt les traces possibles d'une "nature humaine », qui rend possible une poétique de la volonté au sein du monde naturel et humain. Enfin, pour conclure, nous émettrons l'hypothèse selon laquelle la compréhension du concept de nature à l'aune du « sentir » et de l' « agir », chez MerleauPonty comme chez Ricœur, fait signe vers une refondation de la métaphysique du sujet à même de tenir ensemble les perspectives cosmologique et anthropologique.

\footnotetext{
${ }^{11}$ P. Ricœur, « Devenir capable, être reconnu », in Esprit, n ${ }^{\circ}$ 7, juillet 2005, p. 125.

12 « Par le biais du problème de la disproportion et de l'intermédiaire, une refonte de la notion de finitude devient possible. Pour le dire tout net, je doute que le concept de finitude soit le concept central de l'anthropologie philosophique - mais bien plutôt la triade finitude-infinitude-intermédiaire. », P. Ricœur, «L'antinomie de la réalité humaine et le problème de l'anthropologie philosophique », in Anthropologie Philosophique, Écrits et conférences 3, Paris, Seuil, 2013, p. 23.

${ }^{13}$ M. Merleau-Ponty, La Nature, Cours du Collège de France, Paris, Seuil, 1995, p. 19.
} 
Mythe(s) de l'intériorité et phénoménologie de la nature

\section{Nature et Individuation}

«Il faut que la pensée de science - pensée de survol, pensée de l'objet en général - se replace dans un "il y a" préalable, dans le site, sur le sol du monde sensible et du monde ouvré tels qu'ils sont dans notre vie ", écrit Merleau-Ponty dans L'œil et l'esprit ${ }^{14}$. Cette exhortation achève la mise au jour réalisée par la Phénoménologie de la perception: les présomptions de la raison objective - impliquées autant par une perspective empiriste que par une démarche intellectualiste - doivent être démasquées pour laisser apparaître une situation plus originaire. Repenser «notre » situation revient pour Merleau-Ponty à retracer les contours d'un certain mode d'être au monde, esquissé par la vie préréflexive de la pensée en tant qu'elle est incarnée et qu'elle est au monde, avec d'autres, avant même d'être engagée dans un rapport théorique d'objectivation. Retourner au fondement, " au sol du monde sensible et du monde ouvré » ne signifie pas pour Merleau-Ponty se mettre en quête d'une nouvelle raison suffisante qui viendrait légitimer une vérité préalablement établie par un certain régime de pensée. Le caractère radical ainsi visé correspondrait plutôt à la démarche phénoménologique elle-même. Cette dernière tend à renouer avec ce sol originaire, avec cette facticité ${ }^{15}$ primordiale du monde sensible, qui préexiste à toutes nos réflexions ; elle cherche à faire sens de l'évidence de l'absence de fondement, à faire jaillir une certaine positivité de l'indéterminé. Ce retour à la facticité primordiale opéré par la Phénoménologie de la perception, anticipe la caractérisation merleau-pontienne de la nature, qui se profile dans les années cinquante, puisqu'il brise les fondements métaphysiques de la dialectique du pour-soi et de l'ensoi, de l'intériorité et de l'extériorité, mobilisés jusqu'alors pour caractériser les domaines de la liberté et de la nature. Renvoyant dos à dos matérialisme et idéalisme, Merleau-Ponty constate : "tandis que le corps devenait un extérieur sans intérieur la subjectivité devenait un intérieur sans extérieur, un spectateur impartial $»^{16}$.

\footnotetext{
14 M. Merleau-Ponty, L'oil et l'esprit, Paris, Gallimard, 2002, p. 12.

15 « Mais la phénoménologie, c'est aussi une philosophie qui replace les essences dans l'existence et ne pense pas qu'on puisse comprendre l'homme et le monde autrement qu'à partir de leur "facticité". [...] «c'est aussi une philosophie pour laquelle le monde est toujours "déjà là" avant la réflexion, comme une présence inaliénable et dont tout l'effort est de retrouver ce contact naïf avec le monde pour lui donner enfin un statut philosophique. », M. Merleau-Ponty, Phénoménologie de la perception, op. cit., p. i.

${ }^{16}$ M. Merleau-Ponty, Phénoménologie de la perception, op. cit., p. 68.
} 
Or, selon Merleau-Ponty, rechercher la loi et les raisons, la fin ou l'idée, revient à manquer la contingence de l' «être sauvage» en sa naturalité, revient à

oublier qu'il est l'être à distance, l'attestation fulgurante ici et maintenant d'une richesse inépuisable, que les choses ne sont qu'entr'ouvertes devant nous, dévoilées et cachées : de tout cela, on rend compte aussi mal en faisant du monde une fin qu'en le faisant idée. La solution, - si solution il y a-ne peut être que d'interroger cette couche du sensible, ou de nous apprivoiser à ses énigmes ${ }^{17}$.

Dépasser la dialectique du pour-soi et de l'en-soi exige donc une reformulation du problème de l'articulation de la nature et de la liberté à partir d'une nouvelle approche du « sensible » et du « sentir ». Interroger cette " couche du sensible » ne consiste pas à recouvrir la nature elle-même d'un "vêtement d'idées » ${ }^{18}$, selon l'expression de Husserl. Dépasser la dialectique de l'en-soi et du pour-soi conduit MerleauPonty à mettre au jour une générativité, une morphogenèse du sens, qui dépasse tout à la fois le principe du mécanisme et le principe de finalité ${ }^{19}$ puisqu'il s'agit d'une expressivité qui procède à partir de son propre mouvement phénoménalisant. Telle est en effet, la définition donnée par Merleau-Ponty au début de son Cours sur la Nature:

Il y a nature partout où il y a une vie qui a un sens, mais où, cependant, il n'y a pas de pensée; d'où la parenté avec le végétal : est nature ce qui a un sens, sans que ce sens ait été posé par la pensée. C'est l'autoproduction d'un sens. [...] La Nature est donc différente d'une simple chose; elle a un intérieur, se détermine $d u$ dedans; d'où l'opposition de "naturel» à "accidentel». Et cependant la Nature est différente de l'homme; elle n'est pas instituée par lui, elle s'oppose à la coutume, au discours ${ }^{20}$.

\footnotetext{
${ }_{17}$ M. Merleau-Ponty, Signes, Paris, Gallimard, 2008, p. 273.

18 «Le vêtement d'idées: "Mathématique et science mathématique de la nature", ou encore le vêtement de symboles, de théories mathématico-symboliques, comprend ce qui, pour les savants et les hommes cultivés, se substitue (en tant que nature "objectivement réelle et vraie") au monde de la vie et le travestit. », E. Husserl, La crise des sciences européennes, trad. G. Granel, Paris, Gallimard, 1989, p. 60.

${ }^{19}$ Renaud Barbaras note ici la similitude entre l'analyse de Merleau-Ponty et celle de Bergson: « comme Bergson l'avait déjà montré, [la vie] se situe par-delà l'alternative du mécanisme et du finalisme (elle n'est pas mécanisme puisque l'organisme est polarisé par l'avenir et donc plus que lui-même, mais elle ne relève pas de la finalité car ce qui dynamise le vivant n'est pas un être transcendant et positif, car le devenir du vivant est tributaire de chacune des étapes effectives). Le déploiement de l'animal, dit magnifiquement Merleau-Ponty, est "comme un pur sillage qui n'est rapporté à aucun bateau". », in "Merleau-Ponty et la nature », Chiasmi International, vol. 2, 2000, p. $47-61$.

${ }_{20}$ M. Merleau-Ponty, La Nature, cours au Collège de France, op. cit., p. 19.
} 
Le recours à la notion d'intentionnalité opérante (ou intentionnalité fungierende) telle qu'elle est reprise par Merleau-Ponty permet d'élucider cette définition de la nature comme « une vie qui a un sens ». La nature est à la fois "être sauvage ", échappant aux lois de l'entendement et l' «être interrogatif » qui travaille la vie de l'intérieur en générant et recréant sans cesse ses possibilités d'existence.

Cet être sauvage ou être brut est à la fois immémorial et inaugural. Il est immémorial puisqu'il est toujours déjà là et que la structure temporelle des rythmes de la nature n'obéit pas aux mêmes lois que celles qui régissent la conscience humaine du temps. Il serait ainsi inapproprié de parler d'un commencement de la nature. Cet être brut est pourtant doté d'une force inaugurale puisque ses dynamiques de transformation et les possibilités qu'il recèle permettent à chaque fois une naissance et une co-naissance des singularités. Retrouver cet être brut dans le cadre d'une ontologie - qui ne pourra être qu'indirecte permet de réfuter autant la perspective mécaniste que la perspective finaliste et de repenser l'humain, non à partir de la subjectivité mais à partir d'une nature qui l'ancre et lui donne consistance dans le monde de la vie :

La Nature ne nous intéresse donc ni pour elle-même, ni comme un principe universel d'explication, mais comme index de ce qui dans les choses résiste à l'opération de la subjectivité libre et comme accès concret au problème ontologique. Si l'on refusait tout sens philosophique à l'idée de Nature et si l'on réfléchissait directement sur l'être, on risquerait de se placer d'emblée au niveau de la corrélation sujet-objet qui est élaborée et seconde et de manquer une composante essentielle de l'être: l'être brut ou sauvage qui n'a pas encore été converti en objet de vision ou de choix. C'est lui que nous voudrions retrouver ${ }^{21}$.

L'être sauvage de Merleau-Ponty ne peut être découpé ou saisi par un regard objectivant. Ni pur Être, ni pure matière, la Nature dont il est ici question se situe précisément à l'endroit même où s'articulent les visibles. Comme l'écrit Merleau-Ponty: «tout ce qui se passe ne s'explique pas par l'intériorité, ni par l'extériorité, mais par une chance, qui est la concordance entre ces deux données, et qui est assurée par la Nature $»^{22}$. L'idée de chance renvoie à cette autoproduction du sens évoquée plus haut, et qui se situe à équidistance de la causalité du mécanisme et de la nécessité du finalisme. La porosité de cet être brut permet de repenser l'humanité à partir de sa nature primordialement charnelle et d'y retrouver les trajectoires possibles de sa liberté. Le

${ }^{21}$ M. Merleau-Ponty, in La Nature ou le monde du silence, op. cit., p. 53.

${ }^{22}$ M. Merleau-Ponty, La Nature, cours au Collège de France, op. cit.,p. 12. 
renversement est complet. Il ne s'agit plus d'humaniser la nature selon la logique anthropomorphique du finalisme ou de réifier l'humanité selon la logique naturaliste des sciences exactes mais plutôt « de saisir l'humanité d'abord comme une autre manière d'être corps - de voir émerger l'humanité aussi comme Être en filigrane, non comme une autre substance, comme inter-être et non comme imposition d'un pour soi à un corps en soi $»^{23}$.

Ainsi, la nature et l'histoire n'appartiennent pas à des régimes ontologiques distincts mais correspondent à deux modes irréductibles $\mathrm{d}^{\prime}$ individuation de l'être charnel ${ }^{24}$. Comme l'indique Jacques Garelli dans son Introduction au Logos du Monde esthétique: "C'est alors qu'à l'architectonique réflexive, conçue comme "théorie transcendantale de la méthode », Merleau-Ponty substitue une " architectonique des configurations », par-delà les concepts traditionnels de la conscience, de projection, d'en-soi ou d'objet $»^{25}$. L'histoire et la nature apparaissent alors comme deux configurations possibles de cette logique du sensible qui ouvre la chair au monde et fonde leur intimité26. Lorsque Merleau-Ponty évoque le corps et les sens dans le Visible et l'Invisible comme " mesurants » de l'être, il fait appel à une dimension presque organique du jugement, puisque celui-ci s'élaborerait à l'intersection de l'intercorporéité et du monde naturel. Il ne s'agirait dès lors plus d'un processus de constitution mais plutôt d'une morphogenèse du sens qui serait la condition de possibilité d'une institution qui lui serait coextensive, d'une "institution » qui ne séparerait pas de manière artificielle la vie de la pensée et la pensée de la vie, les animations de la chair et les projections symboliques qui les révèlent dans des cultures ou des situations artistiques ou sociales ${ }^{27}$. La continuité ici affir-

\footnotetext{
${ }^{23}$ M. Merleau-Ponty, La Nature, cours au Collège de France, op. cit.,p. 269.

24 «Ce qu'il y a de commun à l'histoire et à la nature, c'est que ce sont des individuations - mais justement pour cette raison elles sont irréductibles - l'individuation historique est irréductible. », M. Merleau-Ponty, L'institution. La passivité. Notes de cours au Collège de France (1954-1955), op. cit.,p. 40.

${ }^{25} \mathrm{~J}$. Garelli, Introduction au logos du monde esthétique. De la chôra platonicienne au schématisme transcendantal et à l'expérience phénoménologique du monde, Paris, Beauchesne, 2000, p. 372.

${ }^{26}$ « Parce qu'elle nous situe au-delà de l'alternative entre nature et humanité, la chair avoue une double vocation, réellement contradictoire ; elle est naturante autant qu'humanisante - ou plus radicalement encore : humanisante parce que naturante, restituante parce que destituante. Le propre et l'étranger, l'humain et le naturel ne veulent plus faire alternative : la chair est en nous un principe barbare qui voudrait être l'origine de toute raison, une force étrangère qui voudrait nous approprier à nous-mêmes. Cette double dimension de la chair nous semble pouvoir se déduire de la manière dont Merleau-Ponty se réapproprie, sur ce point, l'héritage husserlien. ", É. Bimbenet, Nature et Humanité. Le problème anthropologique dans l'œuvre de Merleau-Ponty, Paris, Vrin, 2004, p. 267.

${ }^{27}$ Cf R. Gély, Imaginaire, perception, incarnation. Exercice phénoménologique à partir de Merleau-Ponty, Henry et Sartre, Bruxelles, Peter Lang, 2012.
} 
Mythe(s) de l'intériorité et phénoménologie de la nature

mée n'est pas l'indice d'une uniformité. Elle révèle la libre conjonction des configurations au sein du monde vécu. Une telle perspective conduit Merleau-Ponty à définir l'humanisme de la manière suivante :

\begin{abstract}
Un humanisme aujourd'hui n'oppose pas à la religion une explication du monde : il commence par la prise de conscience de la contingence, il est la constatation continuée d'une jonction étonnante entre le fait et le sens, entre mon corps et moi, moi et autrui, ma pensée et ma parole, la violence et la vérité, il est le refus méthodique des explications, parce qu'elles détruisent le mélange dont nous sommes faits, et nous rendent incompréhensibles à nous-mêmes ${ }^{28}$.
\end{abstract}

Le primat de la contingence affirmé par Merleau-Ponty, en lieu et place du déterminisme naturel ou d'une liberté émancipée de son ancrage sensible, a le mérite de dépouiller la représentation moderne de l'être-au-monde de ses dualismes. Mais peut-on encore penser la liberté dans un tel contexte et la nature est-elle encore nature si un processus de constitution se donne à voir en elle?

\title{
II. Nature et Intériorité : la personne humaine
}

Dans un article intitulé : « Réhabilitation de l'intériorité. Réflexions sur la dernière philosophie de Merleau-Ponty », publié dans la Revue Philosophique de Louvain en 1986, Stephan Strasser analyse les notionsclés du Visible et l'Invisible et s'interroge sur le bien-fondé du rejet merleau-pontien de la notion d'intériorité et la pertinence d'une pensée de l'Être comme Être du monde qui ne ferait pas droit au partage de l'intériorité et de l'extériorité : « Est-ce que l'Être est coextensif avec l'Être du monde ? Une Extériorité, écrite avec un E majuscule, est-elle pensable? Peut-on parler en philosophie d'une Intériorité qui correspondrait logiquement et répondrait de façon existentielle à cette Extériorité transcendante ? $»^{29}$ Selon Strasser, Merleau-Ponty aurait nié la notion d'intériorité. Cette négation repose, selon Strasser, sur une triple dénonciation : 1/ le rejet de l'intériorité cartésienne, présentée comme «res cogitans »; 2 / le rejet de la conscience transcendantale husserlienne, même si, dans la Phénoménologie de la perception et dans Psychologie et Pédagogie de l'Enfant, Merleau-Ponty s'était appuyé sur la phénoménologie husserlienne pour critiquer la psychologie introspective ; $3 /$ le rejet de la conscience sartrienne, c'est-à-dire d'une «inté-

\footnotetext{
${ }^{28}$ M. Merleau-Ponty, « L'homme et l'adversité », in Signes, op. cit., p. 393.

${ }^{29} \mathrm{~S}$. Strasser, «Réhabilitation de l'intériorité. Réflexions sur la dernière philosophie de MerleauPonty ", in Revue philosophique de Louvain, tome 84, n 64, 1986, p. 503.
} 
riorité qui n'existe que dans la mesure où elle est négation et manque $»^{30}$. Si le «sujet est pure négativité, il ne saurait appartenir à la réalité du monde $»^{31}$. Les notions merleau-pontiennes de réversibilité, de chair, d'entrelacs et d'intercorporéité seraient ainsi les principaux outils de cette triple critique de l'intériorité. Selon Strasser :

Le dernier Merleau-Ponty réagit de façon radicale contre le spiritualisme traditionnel, contre l'idéalisme phénoménologique et contre le négativisme de l'Être et le néant. Sa réaction est non seulement compréhensible, elle contient aussi des idées nouvelles et précieuses pour l'anthropologie phénoménologique; seulement il va trop loin ${ }^{32}$.

En effet, selon Strasser, la notion d'expression, telle que MerleauPonty la développe dans sa phénoménologie du langage et son esthétique $^{33}$ présuppose la notion d'intériorité. « Non que l'idée résiderait toute prête dans un "for intérieur" et ne serait transportée dehors que grâce à un deuxième acte, l'acte expressif $»^{34}$. Mais, nous dit Strasser, «la question qui nous intrigue, c'est la possibilité du mensonge. Si l'expression était vraiment l'occupation de "flotter dans l'Être avec une autre vie, se faire le dehors de son dedans et le dedans de son dehors" (VI, 189), personne ne saurait feindre, tromper, jouer la comédie $»^{35}$. Strasser se demande alors si l'intériorité et l'extériorité d'une personne sont parfaitement réversibles. Il s'interroge sur le caractère inaliénable et irréductible de l'intériorité éthique en abordant notamment le phénomène de la honte ou le sentiment de responsabilité et en questionnant le statut de la réflexivité et de la motivation dans le contexte merleau-pontien. Selon Strasser, la dernière philosophie de MerleauPonty est une "théorie du monde», qui décrit "le logos du monde esthétique », sans jamais pouvoir rendre compte des motivations de l'agir éthique, car elle évacue le principe d'une séparation radicale du même et de l'autre, pourtant aux sources du discours éthique :

Ce qui manque à la théorie du monde telle qu'elle se présente dans la dernière philosophie de Merleau-Ponty, c'est la catégorie de la personne, catégorie indispensable en éthique, en philosophie sociale et en philosophie du droit. Husserl en parle abondamment (Ideen II, § 47-61) Pour rendre

\footnotetext{
${ }^{30} \mathrm{Idem}$.

${ }^{31}$ Idem.

32 S. Strasser, «Réhabilitation de l'intériorité. Réflexions sur la dernière philosophie de MerleauPonty », art.cit., p. 508.

33 Voir par exemple M. Merleau-Ponty, «Sur la phénoménologie du langage » (1951), in Signes, op. cit.

${ }^{34}$ S. Strasser, « Réhabilitation de l'intériorité. Réflexions sur la dernière philosophie de MerleauPonty », art.cit., p. 509.

35 Ibid.
} 
Mythe(s) de l'intériorité et phénoménologie de la nature

compte de l'identité des choses, Merleau-Ponty nous renvoie à une "pulpe spatiale et temporelle où les individus se forment par différenciation » (VI, 153). Mais il devrait ajouter que cette thèse ne saurait être appliquée aux individus humains, porteurs d'une responsabilité éthique, sociale et juridique, unique et personnelle ${ }^{36}$.

Enfin, selon Strasser, les thèses de l'adhérence au monde de la vie et de la foi perceptive ne rendent pas compte de ce que le philosophe allemand nomme, à la suite de Schopenhauer, le «besoin métaphysique de l'homme »: " ces grands penseurs n'ont pas remarqué que la foi perceptive est toujours contrecarrée par une certaine incroyance [...]. L'incroyance dont nous parlons est aussi fondamentale que la foi perceptive. Elle est motivée par la conviction que ce qui se présente comme réel dans le monde visible et tangible n'est pas la réalité ultime ${ }^{37}$. Strasser nomme cette " incroyance », " foi métaphysique », par opposition à la « foi perceptive». Elle rend nécessaire pour lui une phénoménologie de l'intériorité, capable de rendre compte du caractère irréductible de cette « foi métaphysique » et du seuil ontologique qu'elle met au jour :

Merleau-Ponty reproche aux Cartésiens d'avoir dévalorisé le monde clos au profit d'un infini positif. Lui-même opte pour un "infini Offenheit » et rejette un "infini Unendlichkeit»(VI, 223). Mais alors le lecteur est tenté de s'exclamer: de deux choses l'une. Ou bien l'ouverture de l'esprit humain, sa Offenheit, est infinie, et alors elle transcendera la "cloison" du monde; ou bien l'esprit humain est essentiellement mondain, et c'est cela que MerleauPonty semble suggérer en accentuant la "Weltlichkeit du Geist» (VI, 228) et alors son ouverture sera nécessairement limitée $e^{38}$.

Autrement dit, la refonte de la perception dans une phénoménologie de la nature, devenue endo-ontologie de la chair du monde, dissout, purement et simplement, selon Strasser, la question de la transcendance et de la liberté. Pour Strasser, la corrélation entre la foi métaphysique et la foi perceptive, est le propre même du désir. Il suggère $d$ 'ailleurs pour conclure son article que Merleau-Ponty aurait entrevu cette réhabilitation, cette reconfiguration de l'intériorité par le désir et cite le passage suivant du Visible et l'Invisible :

Nous interrogeons notre expérience, précisément pour savoir comme elle nous ouvre à ce qui n'est pas nous. Il n'est même pas exclu par là que nous

\footnotetext{
${ }^{36} \mathrm{~S}$. Strasser, « Réhabilitation de l'intériorité. Réflexions sur la dernière philosophie de MerleauPonty », art.cit., p. 511.

${ }^{37}$ S. Strasser, « Réhabilitation de l'intériorité. Réflexions sur la dernière philosophie de MerleauPonty », art.cit., p. 513.

${ }^{38} \mathrm{~S}$. Strasser, « Réhabilitation de l'intériorité. Réflexions sur la dernière philosophie de MerleauPonty », art.cit., p. 516.
} 
trouvions en elle un mouvement vers ce qui ne saurait être présent en original et dont l'absence irrémédiable compterait ainsi au nombre des expériences originaires. » (VI, 211). Ces mots donnent à penser. N'est-ce pas précisément l'absence irrémédiable et douloureuse de l'Infini qui évoque le « désir»? Merleau-Ponty serait-il malgré tout un métaphysicien" ?

Dans cet article, Strasser oppose donc à l'ontologie merleau-pontienne de la "chair du monde», ce qu'on pourrait nommer une «phénoménologie de l'intériorité ». Celle-ci viserait à ressaisir les traces possibles d'une "nature humaine", irréductible aux individuations des mondes naturel et historique. Selon Strasser, l'enjeu d'une interrogation du concept de nature réside moins dans une requalification de la topologie cartésienne de la res extensa que dans une compréhension des racines de l'affectivité humaine. C'est à présent vers la philosophie de la volonté de Ricœur, citée en exemple par Strasser dans ce même article et qualifiée de "philosophie de l'intériorité » que nous souhaiterions nous tourner.

Aux thèmes de l'écart et de l'absence qui creusent le monde perçu et figurent la question du désir dans la dernière philosophie de Merleau-Ponty, répondent les thèmes de la disproportion et de la faillibilité de l'agir humain lié à sa fragilité affective, dans la philosophie de Ricœur. Ainsi, chez Ricœur, il s'agit de penser l'unité immanente à l'agir humain sans pour autant ignorer une certaine opacité de la conscience à elle-même, née des dimensions de l'involontaire qui surgissent à même la corporéité. Mais comme chez Merleau-Ponty, pensant l'unité impérieuse de la nature comme autoproduction et morphogenèse, l'unité immanente à l'agir humain, dont il est question chez Ricœur est «en devenir» et se déprend d'une pensée de la substance pour mettre en avant une reconfiguration de la métaphysique de la puissance et de l'acte ${ }^{40}$. La philosophie de la volonté déployée par Ricœur possède donc une visée similaire à la démarche merleaupontienne, mais en partant cette fois de l'intériorité. Il ne s'agit pas de penser l'intériorité intrinsèque à la nature mais plutôt la nature - ou les dimensions naturelles qui fomentent ce que Ricœur va nommer l'intimité ou la passivité intime. Comme nous allons le voir, la question de l'intériorité soulevée par Strasser permet de rendre compte d'un dialogue entre Merleau-Ponty et Ricœur concernant la notion de nature humaine. La question de l'intériorité se déploie au sein d'une anthro-

\footnotetext{
${ }^{39}$ S. Strasser, « Réhabilitation de l'intériorité. Réflexions sur la dernière philosophie de MerleauPonty », art.cit, p. 520.

${ }^{40}$ Voir P. Ricœur, «Vers quelle ontologie ? », in Soi-même comme un autre, Paris, Seuil, 1990, p. 345410.
} 
pologie philosophique qui vise à saisir de manière nouvelle les fondements de la personne en ne dissociant pas le for intérieur - essentiel à la réflexion éthique - de sa chair affective et intersubjective : "C'est dans ces échanges entre tensions vitales et requêtes du "soi" d'une part, requête du "soi" et appétit de bonheur d'autre part, que se "médiatise" la vie de la personne ${ }^{41}$. Cette "médiatisation " s'opère sous l'effet d'une symbolisation des affects et d'une métaphoricité du réel qui n'est pas sans faire écho à la la conception merleau-pontienne de l'institution symbolique. Ainsi, la philosophie de l'intériorité proposée par Ricœur enracine la liberté et la volonté dans le corps et la vie :

Une certaine tradition intellectualiste croit devoir sauver l'originalité de la volonté en opposant les motifs aux mobiles; les mobiles seraient affectifs et passionnels, les motifs rationnels et assagis. [...] Il faudra pourtant se convaincre que la plupart de nos motifs ne sont pas faits d'une autre étoffe que notre vie affective; toute notre conception du corps, de l'involontaire corporel offert au magistère du "Je veux», reposera sur cette conviction que c'est l'élan même de l'involontaire corporel qui meut notre vouloir, mais d'une motion sui generis que notre arbitre adopte en se décidant ${ }^{42}$.

Là où la liberté s'exprimait dans le vivant chez Merleau-Ponty, la vie s'exprime dans la liberté chez Ricœur : «La vie n'est pas seulement la partie basse de moi-même sur laquelle je règne ; je suis vivant tout entier, vivant dans ma liberté même. Je dois être en vie pour être responsable de ma vie. Cela que je commande me fait exister $»^{43}$. La dialectique de l'en-soi et du pour-soi est ici raturée par une pensée de l'involontaire qui fait échouer autant le mécanisme que le finalisme anthropomorphique. Au mécanisme qui réduit l'espace à l'immanence des mouvements qui le structurent, et au finalisme qui cloisonne l'horizon du temps dans le déterminisme imposé par le fondement qu'il cherche à justifier, Ricœur oppose une conception de la contingence qui n'oppose plus la nature et la grâce et qui fait de la liberté le signe d'une ipséité proprement charnelle : «Ainsi, j'éprouve sans cesse en moi le mélange de deux involontaires : l'involontaire absolu d'une vie qui me donne d'exister comme conscience - et ainsi préface mon humanité, - et l'involontaire relatif d'une vie qui sollicite ma décision et mon effort, et ainsi attend mon humanité $»^{44}$. Dans la pensée de Ricœur, comme dans celle de Merleau-Ponty, se joue in fine la refor-

\footnotetext{
${ }^{41} \mathrm{P}$. Ricœur, «L'antinomie de la réalité humaine et le problème de l'anthropologie philosophique », op. cit., p. 45.

${ }_{42}$ P. Ricœur, Philosophie de la volonté 1, le volontaire et l'involontaire, op.cit, p. 199.

${ }^{43}$ Ibid., p. 513.

${ }^{44}$ Ibid., p. 525.
} 
mulation de l'anthropologie à l'aune d'une conception renouvelée de la cosmologie (Merleau-Ponty) ou de la métaphysique du sujet (Ricœur). Sans quitter l'ancrage sensible de la subjectivité, il convient alors de repenser l'humanisme à l'aune d'une nature qui est moins l'autre de l'homme que le corps de son plein épanouissement.

Bien qu'empruntant des trajectoires adverses, les réflexions de Merleau-Ponty et de Ricœur sur la nature aboutissent à une remise en question et à une reformulation de l'intériorité et partant, du rapport de la pensée humaine elle-même à la nature qui lui permet d'exister et de se formuler. Là où Merleau-Ponty cherche, dans les dernières notes du Visible et l'Invisible, à ancrer le sentiment et la dimension spirituelle de l'existence dans la corporéité et dans une approche esthésiologique de la réalité, Ricœur choisit d'ancrer la vie et son dynamisme dans le sentiment qui l'arrache à l'immédiateté de l'auto-affection qui la caractérise. Ainsi, comme l'écrit Ricœur :

\begin{abstract}
La fonction universelle du sentiment est de relier; il relie ce que la connaissance scinde; il me relie aux choses, aux êtres, à l'être [...]. Mais en intériorisant toutes les liaisons du moi au monde il suscite une nouvelle scission, de soi à soi. Il rend sensible la dualité de la raison et de la sensibilité qui trouvait dans l'objet un point de repos; il distend le moi entre deux visées affectives fondamentales, celle de la vie organique qui s'achève dans la perfection instantanée du plaisir, celle de la vie spirituelle qui aspire à la totalité, à la perfection du bonheur ${ }^{45}$.
\end{abstract}

Dans ces lignes de Ricœur se profile la dialectique de la passivité et de l'activité qui meut l'homme toujours solidairement "agissant et souffrant». Le sentiment est tout à la fois ce qui relie l'homme au monde et ce qui lui permet de faire l'expérience de la faillibilité et de l'intransitivité de son désir. Il est, en un sens, la marque de cet écart qui rend possible la constitution d'une nature comme nature humaine. Les dernières notes du Visible et l'Invisible font signe, quant à elle, vers une reconfiguration métaphysique de l'intériorité : "par principe le côté "spirituel" d'un corps vivant ne peut m'être selbstgegeben que comme absence » $[. .$.$] « après nature physique et vie, faire un 3^{\text {e }}$ chapitre où le corps humain sera décrit comme ayant un côté "spirituel" ${ }^{46}$. Ces réflexions amorcent le projet merleau-pontien d'une nouvelle topographie de la liberté, ancrée dans le sensible et indissociable d'une nature qui serait alors l'autre nom de la corporéité et du vivant. Merleau-Ponty vise ici à dépasser le partage de l'empirique et du trans-

\footnotetext{
${ }^{45}$ P. Ricœur, Philosophie de la volonté 2, Finitude et Culpabilité, op. cit., p. 182.

${ }^{46}$ M. Merleau-Ponty, note de janvier 1959, Le visible et l'invisible, op. cit., p. 220.
} 
Mythe(s) de l'intériorité et phénoménologie de la nature

cendantal, pour mieux déployer une anthropologie phénoménologique que l'on pourrait qualifier $\mathrm{d}^{\prime}$ ' intégrale » et qui prend ses distances avec la séparation de la nature et de la liberté en deux régimes distincts, au profit d'une conception renouvelée de l'affectivité. Mais comme le souligne Fabrice Colonna : «L'échec de Merleau-Ponty tient peut-être à ce qu'il a recherché l'impossible : une métaphysique - réaffirmée et défendue - mais d'un point de vue finitiste ou phénoménologique. C'était peut-être là un oxymore, correspondant à l'impossibilité que Paul Ricœur diagnostiquait dans les "cosmologies », qui veulent tenir à la fois le Cogito et la Nature $»^{47}$. La réflexion de Ricœur nous invite, quant à elle, à penser solidairement la praxis d'une liberté incarnée et la passivité radicale qui fait de sa sensibilité la trace d'une nature irréductible à la perception. Ainsi :

Dans une dialectique acérée entre praxis et pathos, le corps propre devient le titre emblématique d'une vaste enquête qui, au-delà de la simple mienneté du corps propre, désigne toute la sphère de passivité intime, et donc de l'altérité, dont il constitue le centre de gravité. Il faudrait, dans cette perspective, parcourir le travail conceptuel qui s'est fait depuis les Traités classiques des passions, en passant par Maine de Biran, jusqu'à la méditation de Gabriel Marcel, de Merleau-Ponty et de Michel Henry, sur l'incarnation, la chair, l'affectivité et l'auto-affection ${ }^{48}$.

L'approche phénoménologique et herméneutico-morale des problèmes de la liberté et de la nature esquisse ainsi une reformulation du partage du transcendantal et de l'empirique au profit d'une configuration "hyper-dialectique» de leurs rapports. Cette perspective ne renie ni la perception incarnée qui esquisse le réel ni la souveraineté d'une vie qui fonde son ipséité dans la communication intime de son appartenance au monde et de sa différence à soi. Ainsi, la compréhension du concept de nature à l'aune du « sentir » et de l' « agir », chez Merleau-Ponty comme chez Ricœur, fait signe vers une refondation de la métaphysique du sujet à même de tenir ensemble les perspectives cosmologique et anthropologique qu'il porte sur la nature.

\section{D'une Nature à l'autre : appartenance et médiation}

«La phénoménologie, [...] n'élimine que la métaphysique naïve, opérant avec les absurdes choses en soi, mais elle n'exclut pas la

\footnotetext{
${ }^{47}$ F. Colonna, Merleau-Ponty et le renouvellement de la métaphysique, Paris, Hermann, 2014, p. 451-452.

${ }^{48}$ P. Ricœur, Soi-même comme un autre, op. cit., p. 371.
} 
métaphysique en général $\gg^{49}$ écrivait Husserl à la fin des Méditations cartésiennes. Le dépassement de la dialectique de l'en-soi et du pour-soi n'annule ni l'idée d'intentionnalité, ni la vocation métaphysique de la philosophie qui est « la foi perceptive s'interrogeant sur elle-même. On peut dire d'elle, comme de toute foi, qu'elle est foi parce qu'elle est possibilité de doute, et cet infatigable parcours des choses, qui est notre vie, est aussi une interrogation continuée $»^{50}$. Deux directions s'offrent alors au désir qui sous-tend une telle interrogation sur les rapports de la nature à la liberté : la voie esthétique du sentiment qui, de l'anthropologie à la poétique, exprime la Nature ; la voie théologico-herméneutique de l'intériorité qui, de la théologie négative à l'éthique, est imprimée dans la nature sensible. La porosité du sensible mise en lumière par MerleauPonty permet de penser le caractère coextensif de la Nature et du sens qu'elle génère. La polarité permise par l'intimité fondatrice de l'ipséité du sujet assure l'ancrage charnel d'une liberté qui prend corps dans le monde naturel et humain. Deux risques sont associés à ces deux chemins. La voie esthétique, ou plutôt esthésiologique, pourrait se perdre dans une gnose du sentir qui pourrait refermer l'horizon cosmologique et métaphysique pourtant appelé par une pensée de la nature. Ce risque surgirait dès lors que la description phénoménologique de la porosité se dispenserait d'une reformulation des conditions de possibilité de la réflexivité engagée dans cette tâche. La voie théologico-herméneutique de l'intériorité risquerait, quant à elle, de se perdre dans une mythologie de l'agir qui pourrait refermer l'horizon éthico-pratique d'une pensée du sujet si celle-ci ignorait la radicale passivité à l'œuvre dans la souffrance qui nous rappelle sans cesse notre connaturalité au monde sensible. Ainsi, une reconfiguration phénoménologique du problème de la nature nous inviterait à articuler le régime de porosité qui régit l'être charnel et le régime de polarité qui structure autant sa dimension spirituelle - au sens de la vie de l'esprit - que sa dimension expressive. Se rejoignent ici le thème esquissé par Merleau-Ponty d'une vulnérabilité de l'être qui se fait nature et celui développé par Ricœur d'une vulnérabilité de l'homme « agissant et souffrant » sur laquelle est sise sa nature proprement humaine.

Penser la nature d'un point de vue phénoménologique paraît alors indissociable d'une interrogation sur le monde-qu'il s'agisse du monde naturel ou du monde vécu - et sur le sujet auquel il se donne. La nature se livre sous la forme d'un monde qui fait sens pour le sujet qui la contemple ou la transforme. Telle est peut-être la limite de la

${ }^{49}$ E. Husserl, Méditations cartésiennes, § 64, Paris, PUF, 1990, p. 182.

${ }_{50}$ M. Merleau-Ponty, Le visible et l'invisible, op. cit., p. 137. 
Mythe(s) de l'intériorité et phénoménologie de la nature

démarche phénoménologique - notamment pour la formulation d'une écologie - mais aussi sa ressource propre. Comme le disait Ricœur dans un entretien sur l'éthique et l'écologie :

Lorsque l'homme s'excepte de la nature comme s'il était l'autre de la nature alors, dans le langage de Heidegger, il se met en scène lui-même comme le centre d'un grand spectacle qu'il organise, et aussi d'un chantier inerte de choses à manipuler. [...] Il faut tenir la subtilité de cet équilibre fragile comme à la fois dans la nature et en même temps exception de la nature ${ }^{51}$.

Loin de penser le métaphysique dans l'homme et la question de l'intériorité comme les autres de l'écologie et d'une phénoménologie du monde naturel, il s'agirait plutôt de frayer le chemin à une reformulation conjointe de ces dimensions, à partir d'une anthropologie et d'une cosmologie dévêtues de leurs présupposés strictement rationalistes ou vitalistes. Ainsi, les philosophies de Merleau-Ponty et de Ricœur pensent la nature en dialogue avec l'intériorité - intériorité de l'être ou intériorité du sujet - parce qu'elles s'intéressent aux modes de phénoménalisation de la nature à travers le prisme du vivant. Leurs réflexions sur la nature et sur la liberté s'approfondissent dans une interrogation métaphysique sur les strates et les degrés qui vont de la nature brute et sauvage jusqu'aux produits les plus achevés de la culture et au travers desquels l'homme exprime le "monde de la vie ». Deux concepts paraissent alors illustrer cette dimension active et processuelle de la nature et sa conversion symbolique par l'esprit humain : celui d'appartenance et celui de médiation.

Comme l'indique Renaud Barbaras, Merleau-Ponty

porte au premier plan et tente de penser le phénomène de l'appartenanceque l'on peut spécifier de prime abord comme appartenance du sujet au monde - en tant que condition de la perception. Il n'y a de monde pour un sujet que dans la mesure où celui-ci est du monde. Toute la difficulté est de penser cette appartenance de manière rigoureuse ${ }^{52}$.

Or, Merleau-Ponty, selon Renaud Barbaras, échouerait à penser rigoureusement cette appartenance précisément parce qu'il la résumerait aux modalités d'incarnation du sujet, à son ancrage naturel et charnel. Le sens détermine encore le corps comme tel. La porosité semble ne pas pouvoir se dire sans la polarité qui la laisse voir. Ricœur, quant à lui, s'intéresse à la médiation, c'est-à-dire au point d'articulation

\footnotetext{
${ }^{51}$ P. Ricœur, «L'éthique, le politique, l'écologie. Entretien avec Paul Ricœur [Propos recueillis par Edith et Jean Paul Deléage] ", Écologie politique. Sciences, Culture, Société, n 7, 1993.

${ }^{52}$ R. Barbaras, Entretien avec François-David Sebbah, Rue Descartes, Collège International de Philosophie, $\mathrm{n}^{\circ} 70,2010$, p. 93.
} 
de cette «antinomie constitutive de la réalité humaine » qui est celle $\mathrm{du}$ caractère et $\mathrm{du}$ bonheur - du donné naturel et de la destination éthique de l'homme :

\begin{abstract}
Ainsi, je ne puis penser le caractère et le bonheur que conjointement, comme une antinomie constitutive de la réalité humaine: le caractère, c'est l'orientation perspectiviste du champ total de motivation; le bonheur, c'est le terme vers lequel toute ma motivation est orientée. Point zéro et horizon: ainsi se généralise l'analyse de la perception avec son "point de vue » et son "horizon » du monde [...] L'unité du bonheur et du caractère est une tâche et cette tâche est ce que nous appelons l'idée de personne $e^{53}$.
\end{abstract}

Selon qu'il se formule dans les termes d'une topologie (MerleauPonty), ou dans les termes d'une eschatologie (Ricœur) le sens conféré à la nature affective et sensible de l'être humain appelle son dépassement vers l'horizon symbolique du monde de la vie - de la vie du corps ou de la vie de l'esprit. C'est ce surgissement même du sens, en somme, que ces deux philosophies cherchent à capturer dans ce que Natalie Depraz nomme une « ontologie modale ou aspectuelle ». Là en effet, " le phénomène y est accent, densité, augmentation d'être : ni sa création, ni sa génération, juste un signal léger de sa présence ${ }^{54}$. La nature, comme la chair, se devine dans ses manifestations et ses surrections et ne se donne que de manière "indirecte", comme l'avait pressenti Merleau-Ponty lorsqu'il indiquait vouloir élaborer une "géologie transcendantale » fondant ensemble les structures de l'espace et du temps. Il semble, cependant, comme le montre Ricœur, que ce silence de la nature résulte autant de ses dynamiques propres d'animation que des difficultés rencontrées par l'esprit humain dès lors qu'il cherche à circonscrire sans médiation ce qui, par définition, excède toute pensée. La nature se fait ainsi elle-même mythique et poétique, au-delà des objectivations qui la déterminent. Telle est alors la ligne de crête de l'anthropologie philosophique comme l'aiguillon de sa fécondité: «L'anthropologie philosophique n'est jamais achevée. Et surtout elle n'a jamais fini de récupérer l'irrationalité de sa source non philosophique dans la rigueur de la réflexion. Sa détresse est de ne pouvoir sauver à la fois la profondeur du pathos et la cohérence du logos $»^{55}$.

\footnotetext{
${ }^{53} \mathrm{P}$. Ricœur, «L'antinomie de la réalité humaine et le problème de l'anthropologie philosophique », op. cit, p. 38-39.

${ }_{54}$ N. Depraz, "Attention et surprise. Paul Ricœur en débat et au-delà », Alter, n 23, 2015, p. 277. ${ }^{55} \mathrm{P}$. Ricœur, «L'antinomie de la réalité humaine et le problème de l'anthropologie philosophique », op. cit, p. 46.
} 